\title{
Electronic literary text: cohesion and coherence aspects
}

\author{
Svetlana Kuchina ${ }^{1, *}$ \\ ${ }^{1}$ Novosibirsk State Technical University, 630073, 136 Nemirovicha-Danchenko str., Novosibirsk, \\ Russia.
}

\begin{abstract}
The article deals with the questions of electronic literary text cohesion and coherence specifity. Development of informational and communicational technologies in modern world transformed the text materiality. Nowadays its basic categories relate mostly to the electronic communication features. Electronic text became significant in all spheres of human communication. This factor makes this phenomenon relevant for the research and educational options on the topic. The research materials include several electronic texts (based on different platforms and technologies such as Adobe Flash, Scalar, HTML 5) that demonstrate the use of conceptually valid poly-code elements in their semantic structure. The basic characteristics of the text (printed or electronic) are the categories of cohesion and coherence. The cohesion specifity results from dual (verbal and non-verbal) structure of electronic literary text. The author suggests that the structural aspect of the electronic literary text semantic cohesion is not isolated from the other types of verbal and nonverbal elements correlations because it is not semantic self-contained and complete. The semantic cohesion of electronic literary text poly-code elements is usually represented in both structural and identifying aspects.
\end{abstract}

\section{Introduction}

The article deals with the questions of electronic literary text cohesion and coherence specifity. Development of informational and communicational technologies in modern world transformed the text materiality. Nowadays its basic categories relate mostly to the electronic communication features. Electronic text became significant in all spheres of human communication. This factor makes this phenomenon relevant for the research and educational options on the topic. The most important criteria for electronic literary text defining are its creation, publication and preservation aspects. A piece of electronic literature could not be printed, it is created with and for the electronic medium. N. K. Hayles defines electronic literary texts as texts that have been digitally created or "digital born" [1]. The Electronic Literature Organization's definition of electronic literature emphasizes another distinctive feature of computer-generated creations. The ELO definition is that "the term refers to work with an important literary aspect that takes advantage of the capabilities and contexts provided by the stand-alone or networked

\footnotetext{
* Corresponding author: svkuchina @ yandex.ru
} 
computer" [2]. According to the ELO, the role that the computer plays in the process of creating the text is very important and offers many conceptual, visual and interactive opportunities. However, reading and writing still play an essential role in electronic literature.

The research materials include several electronic texts (based on different platforms and technologies such as Adobe Flash, Scalar, HTML 5) that demonstrate the use of conceptually valid poly-code elements in their semantic structure. The main methods of the research are general scientific methods, such as monitoring and description, in conjunction with the method of comparative analysis and complex linguistic and stylistic analysis of literary text

\section{Electronic literary text cohesion and coherence}

The basic characteristics of the text (printed or electronic) are the categories of cohesion and coherence. The cohesion specifity results from dual (verbal and non-verbal) structure of electronic literary text. Semantic coherence of electronic literary text is based on its verbal and nonverbal elements correlation. This type of correlation is fixed by nonverbal and language means. The language markers of the electronic literary text semantic cohesion can have explicit and implicit character. This type of correlation is also can be defined through the anaphoric and cataphoric location of cohesion means in the electronic text. The author considers the semantic cohesion of electronic literary text elements in structural and identifying aspects.

The structural aspect of semantic cohesion suggests the poly-code elements integration into the verbal structure of electronic literary text. In this regard, nonverbal elements (audio/video/animation/graphics) substitute the corresponding verbal elements (letter/word/sentential unity/chapter) acting as their semantic equivalents. This type of semantic coherence is represented in electronic literary texts with high level of visualization such as interactive dramas and MUD narratives. For example, "Nightingale's playground" electronic narrative by A. Campbell suggests the reader's immersion into 3D reality of the fiction world represented by verbal and nonverbal elements [3]. Visual substitutes of "Nightingale's playground" verbal elements are the interactive images of the electronic narrative such as the window, old suitcase, calculator etc. The text which appears on the screed after these nonverbal elements (interactive images) continues the semantic revealing of the topic initiated by the poly-code elements. Activation of the interactive image of calculator leads to the verbal part of Nightingale's playground appearance on the screen. The thematic aspect initiated by visual component continues in its verbal corresponding element. It becomes clear for the recipient that the calculator is a special sentimental thing, which the main character used in his school days, what is more several notable events in his life are associated with this thing. 


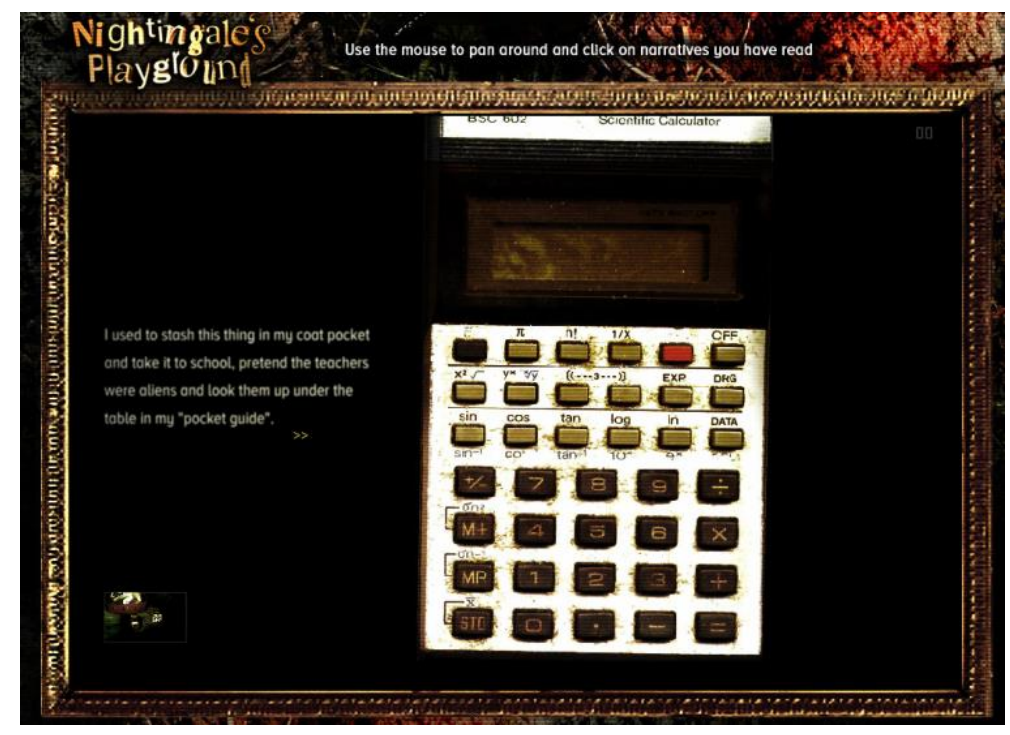

Fig. 1. "Nightingale's playground" by A. Campbell.

The structural aspect of the electronic literary text semantic cohesion is not isolated from the other types of verbal and nonverbal elements correlations because it is not semantic self-contained and complete. The semantic cohesion of electronic literary text poly-code elements is usually represented in both structural and identifying aspects. The identifying aspect of the electronic literary text cohesion is usually used with an appellative purpose. The cohesion implementation is carried out with the help of special language means used in a referential function. Among these language means we can specify personal pronouns, nouns (in nominative function), substantive phrases composed of common nouns, articles, possessive and negative adjectives. Nonverbal elements that are used in the same function can be represented by graphical, audio, video and animation resources. All of them are used in electronic literary texts as semantic equivalents of the corresponding verbal notions.

The identifying aspect of the electronic literary text semantic cohesion have common scheme of representation for all types of electronic narratives. The visual image of the narrative is accompanied by corresponding verbal elements represented by personal pronouns, nouns (in nominative function), substantive phrases composed of common nouns, articles, possessive and negative adjectives. Nonverbal elements that are used in the electronic narrative in this function can be represented by audio, video or graphical image. For example, the character identification in the " 88 Constellations for Wittgenstein" by D. Clark is represented by the correlation of the visual object (the graphical image of L. Wittgenstein) and the audio text about this character (This story is about a man named Wittgenstein. He was a philosopher. His life was a series of moments and our story is a series of constellations). 


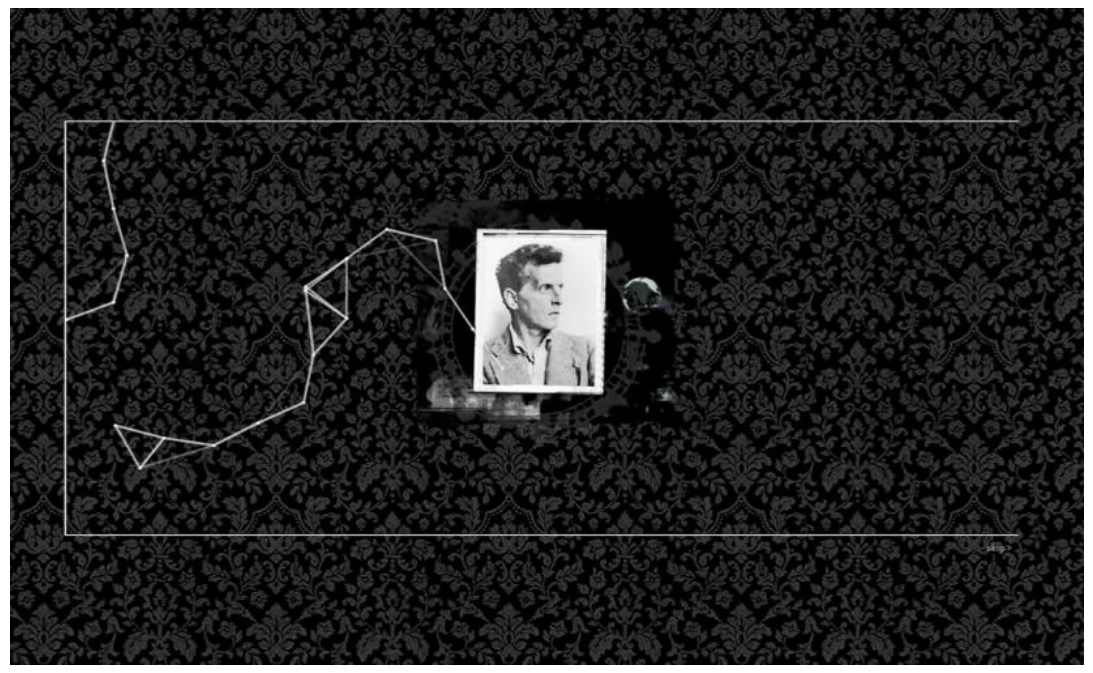

Fig. 2. "88 Constellations for Wittgenstein" by D. Clark.

\section{Conclusion}

The research results allow to suggest that the electronic literary text cohesion on semantic level is represented in structural and identifying aspects. All of them are provided by verbal and nonverbal elements of electronic literary text. The semantic revealing of the electronic literary texts main topics is provided by mental combination of verbal and visual elements in recipient's consciousness during the electronic text perception process. In this case the poly-code paralinguistic elements enhance semantic resources of electronic literary text and emphasize its visibility.

Human conscience has changed a lot since the electronic text permeated all areas of people's life (personal interaction, business, education). It is not a secret that digital native generation needs new ways of information providing and presenting, especially it concerns the entertaining and educational spheres. In this regard, the electronic text is a perfect way to push the limits and boundaries of a training room. It suggests modern, accessible, userfriendly, and gripping resource for training different skills on a multi-disciplinary basis.

\section{References}

1. K. N. Hayles, Electronic literature: what is it? (UCLA, Los Angeles, 2007)

2. Electronic literature organization, Official website (ELO, Vancouver, 2007)

3. A. Campbell, J. Alston, Nightingale's playground (2006)

4. D. Clark, 88 constellations for wittgenstein (ELO, Cambridge, 2018) 